\title{
ON THE RANGE OF REAL COEFFICIENTS OF FUNCTIONS WITH BOUNDED BOUNDARY ROTATION
}

\author{
HEIKKI HAARIO
}

\section{Introduction}

For $k \geqq 2$ let $B_{k}$ denote the class of analytic functions

$$
f(z)=z+a_{2} z^{2}+\ldots
$$

which map the unit disc onto a domain whose boundary rotation is at most $k \pi$. Each function of $B_{k}$ can be obtained as a solution of the equation

$$
1+z \frac{f^{\prime \prime}(z)}{f^{\prime}(z)}=\frac{1}{2} \int_{-\pi}^{\pi} \frac{z+e^{i \varphi}}{z-e^{i \varphi}} d \mu(\varphi),
$$

where $\mu$ is a real Borel measure on $I=(-\pi, \pi]$ satisfying

$$
\int_{-\pi}^{\pi} d \mu=2, \quad \int_{-\pi}^{\pi}|d \mu| \leqq k .
$$

Conversely, every function satisfying (2) belongs to $B_{k}$. For these basic facts of the class $B_{k}$ see for example [7].

The function

$$
f_{k}(z)=\frac{1}{k}\left[\left(\frac{1+z}{1-z}\right)^{k / 2}-1\right]=\sum_{n=1}^{\infty} A_{n}(k) z^{n}
$$

is the solution of (2) when $\mu$ is a measure concentrated on the points $\varphi=0, \varphi=\pi$. The coefficient conjecture for the class $B_{k}$ was $\left|a_{n}\right| \leqq A_{n}(k), n>1$, for all functions (1) in $B_{k}$. The conjecture was proved in the papers [1] and [2].

It is known that every boundary point of a general coefficient body $\left(a_{2}, \ldots, a_{n}\right)$ in the class $B_{k}$ corresponds to a unique extremal function. The function is produced by a measure $\mu$ of finite support with at most $n-1$ positive and $n-1$ negative values $v_{m}, \sum\left|v_{m}\right|=k$, at points $\varphi=\varphi_{m}, m=1, \ldots, 2 n-2$. Integration of (2) with such a 
measure gives the Schwarz-Christoffel representation

$$
f^{\prime}(z)=\prod_{m=1}^{2 n-2}\left(1-t_{m} z\right)^{-v_{m}}, t_{m}=e^{i \varphi_{m}}
$$

for the extremal function $f$, which thus always maps the unit disc onto a polygon [5].

The above proposition gives a general qualitative description of the extremal functions. However, in many specific cases the essential difficulty is caused by the explicit determination of the parameters $t_{m}, v_{m}$. For the first coefficients such explicit information is available.

In order to study the coefficients of functions inverse to functions in $B_{k}$, Kirwan and Shober [4] determined the sharp bounds for the functional $\operatorname{Re}\left\{a_{3}-\alpha a_{2}^{2}\right\}$ ( $\alpha$ real) in $B_{k}$. By aid of this estimate they found for the inverse functions the sharp bound of the third coefficient for all $k \geqq 2$ and the fourth coefficient for $k \geqq 2 \frac{1}{3}$.

The body $\left(a_{2}, a_{3}\right)$ of $B_{k}$ was determined in [3]. The aim of this paper is to find the region of variability of the coefficients $a_{2}, a_{3}, a_{4}$ in the special case of real coefficients. We shall do this by considering a suitable coefficient combination with two real parameters. Our method is quite direct, but some care is needed in choosing the parameters in an effective way. As an application, we get in the real case the maximum of the fourth inverse coefficient also in the interval $2<k<2 \frac{1}{3}$.

\section{The body $\left(a_{2}, a_{3}, a_{4}\right)$ of $B_{k}$ for real coefficients}

Write (2) in the form

$$
1+z \frac{f^{\prime \prime}(z)}{f^{\prime}(z)}=1+\sum_{n=1}^{\infty} c_{n} z^{n}
$$

where the coefficients $c_{n}$ are given by

$$
c_{n}=\int_{-\pi}^{\pi} e^{-i n \varphi} d \mu, \quad n=1,2, \ldots
$$

The initial coefficients of $f$ assume the expressions

$$
\begin{aligned}
2 a_{2} & =c_{1} \\
6 a_{3} & =4 a_{2}^{2}+c_{2} \\
12 a_{4} & =-8 a_{2}^{3}+18 a_{2} a_{3}+c_{3} .
\end{aligned}
$$


For real $a_{2}, a_{3}$ the region $\left(a_{2}, a_{3}\right)$ is given by the inequalities

$$
\begin{gathered}
a_{3} \leqq \frac{2}{3} a_{2}^{2}+\frac{k}{6} \text { for } 2\left|a_{2}\right| \leqq k / 2+1, \\
a_{3} \leqq \frac{2}{3} \frac{k+6}{k-2} a_{2}^{2}+\frac{4}{3} \frac{k+2}{k-2}\left|a_{2}\right|-\frac{3 k+2}{3(k-2)} \text { for } k / 2+1 \leqq 2\left|a_{2}\right| \leqq k,
\end{gathered}
$$

and

$$
\begin{gathered}
a_{3} \geqq \frac{2}{3} a_{2}^{2}-\frac{k}{6} \text { for } 2\left|a_{2}\right| \leqq k / 2-1, \\
a_{3} \geqq \frac{2}{3} \frac{k+6}{k+2} a_{2}^{2}-\frac{4}{3} \frac{k-2}{k+2}\left|a_{2}\right|+\frac{-3 k+2}{3(k+2)} \text { for } k / 2-1 \leqq 2\left|a_{2}\right| \leqq k ;
\end{gathered}
$$

cf. [3]. Figure 1 presents the region $\left(a_{2}, a_{3}\right)$ in the case $k=4$. Equality is reached for $2\left|a_{2}\right| \leqq k / 2+1$ and $2\left|a_{2}\right| \leqq k / 2-1$ by measures with support at the four points $0, \pi / 2, \pi, 3 / 2 \pi$. In the remaining cases equality is obtained by three-jump measures whose support consists of the point 0 or $\pi$ and of two symmetric points $\pm \varphi$ for which $|\cos ( \pm \varphi)| \rightarrow 1$ when $\left|a_{2}\right| \rightarrow k / 2$.

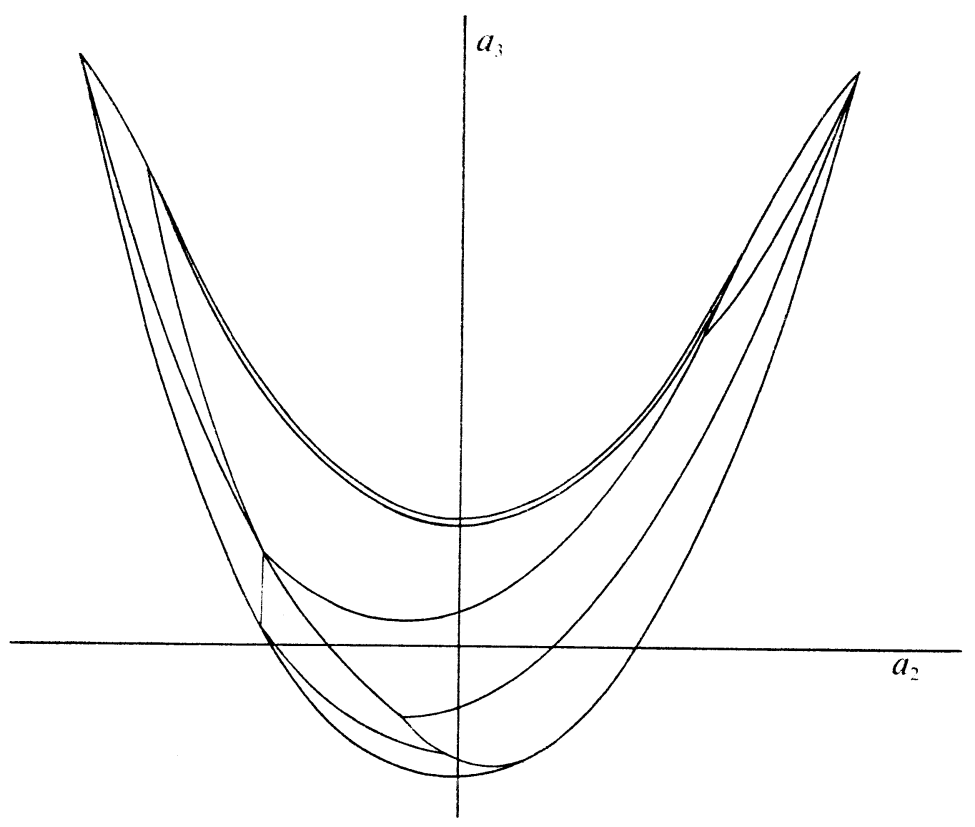

Fig. 1 . 
To determine the real body $\left(a_{2}, a_{3}, a_{4}\right)$ we have to find the extrema of $a_{4}$ for each $a_{2}$ and $a_{3}$ in the body $\left(a_{2}, a_{3}\right)$. We will achieve this by considering a suitable coefficient combination.

Let $t$ and $u$ be real parameters. For real $a_{2}, a_{3}, a_{4}$ the formulae (6) yield

$$
\begin{gathered}
12 a_{4}+8 a_{2}^{3}-18 a_{2} a_{3}+t\left(6 a_{3}-4 a_{2}^{2}\right)+2 u a_{2} \\
=\int_{-\pi}^{\pi}\left(4 \cos ^{3} \varphi+2 t \cos ^{2} \varphi+(u-3) \cos \varphi\right) d \mu-2 t,
\end{gathered}
$$

where we have used the normalization (3). Denote

$$
G(t, u, \varphi)=4 \cos ^{3} \varphi+2 t \cos ^{2} \varphi+(u-3) \cos \varphi,
$$

and decompose the measure $\mu$ into its upper and lower variation, $\mu=\mu^{+}-\mu^{-}$. We should find the maximum of

$$
\int_{-\pi}^{\pi} G(t, u, \varphi) d \mu=\int_{-\pi}^{\pi} G(t, u, \varphi) d \mu^{+}-\int_{-\pi}^{\pi} G(t, u, \varphi) d \mu^{-}
$$

subject to the conditions $\mu^{+}(\mathrm{I})=k / 2+1, \mu^{-}(I)=k / 2-1$.

The integrals with respect to $\mu^{+}$and $\mu^{-}$can be maximized and minimized separately. It follows that the maximum occurs if $\mu^{+}$and $\mu^{-}$are measures supported by sets on which the function $G$ reaches its maximum and minimum, respectively.

Introduce the abbreviation

$$
L=L\left(a_{2}, a_{3}, t, u\right)=\left(-4 a_{2}^{3}+9 a_{2} a_{3}-t\left(3 a_{3}-2 a_{2}^{2}+1\right)-u a_{2}\right) / 6 .
$$

The indentity (7) then yields for the maximum of $a_{4}$ the expression

$$
a_{4}=L+\frac{1}{12} \max _{-\pi<\varphi \leqq \pi} G(t, u, \varphi)\left(\frac{k}{2}+1\right)-\frac{1}{12} \min _{-\pi<\varphi \leqq \pi} G(t, u, \varphi)\left(\frac{k}{2}-1\right) .
$$

The derivative of $G$ with respect to $\varphi$ gives for the points belonging to $\operatorname{spt} \mu$ the possible values $\varphi=0, \varphi=\pi$, or

$$
\cos \varphi=\frac{-t \pm\left(t^{2}-3 u+9\right)^{1 / 2}}{6} .
$$

So, in accordance with the general result mentioned in the introduction, the measure $\mu$ has finite support consisting of (at most) six points. The reality of the integrals $c_{n}, n=1,2,3$ in (6) implies that the values of $\mu$ at the symmetric points given by (9) must be equal. This in turn implies the reality of all $c_{n}, n>3$. 
Observe that different points $t, u$ may produce the same extremal $\mu$ (e.g., all points with $t^{2}-3 u+9<0$ yield the function $f_{k}$ of (4)). Our problem thus reduces to the task of finding for $t, u$ values which yield all the different functions belonging to the boundary of $\left(a_{2}, a_{3}, a_{4}\right)$.

The boundary of $\left(a_{2}, a_{3}, a_{4}\right)$ consists of several parts corresponding to the different types of the measure $\mu$. Since stating a complete theorem which gives all the different parts of the body and the extremal functions would result in a rather massive collection of formulae, we prefer to formulate the equations briefly in the course of the proof. We obtain each part of the body by rewriting the parameters $t, u$ in suitably selected expressions. The specific forms of the expressions were found by considering a few examples.

Our first choice is

$$
\begin{aligned}
& t=-2(2 \alpha+1), \\
& u=4 \alpha^{2}+8 \alpha+3,
\end{aligned}
$$

$-1 \leqq \alpha \leqq 1$. The formula (9) assumes the values $\cos \varphi_{1}=\alpha, \cos \varphi_{2}=(\alpha+2) / 3$. Write the function $G(t, u, \varphi)$ more briefly as $G(\varphi)$. The values of $G$ at the possible points of spt $\mu$ are

$$
\begin{aligned}
G(0) & =G\left( \pm \varphi_{1}\right)=4 \alpha^{2}, \\
G\left( \pm \varphi_{2}\right) & =4(\alpha+2)^{2}(4 \alpha-1) / 27, \\
G(\pi) & =-4 \alpha^{2}-16 \alpha-8 .
\end{aligned}
$$

We can now immediately give the maximum of $a_{4}$ by selecting the largest and smallest among the numbers (11) for different values of $\alpha$. In what follows we use the notation $\delta(\theta)$ for a probability measure with unit mass at the point $\varphi=\theta$.

Let $-1 / 2<\alpha<1$. Since then $G(0)=G\left( \pm \varphi_{1}\right)>G\left( \pm \varphi_{2}\right)>G(\pi)$, we see that the maximum of $a_{4}$ occurs if the mass of $\mu^{+}$is distributed among the points $\varphi=0$ and $\varphi= \pm \varphi_{1}$, and $\mu$ is concentrated on $\varphi=\pi$. That is, the measure $\mu$ consists of the four parts $v \delta(0),(1 / 2)(k / 2+1-v) \delta\left( \pm \varphi_{1}\right)$ and $-(k / 2-1) \delta(\pi)$, where $0 \leqq \nu \leqq$ $k / 2+1$.

Note that there are two free paremeters $\alpha, v$ giving a slice of the body $\left(a_{2}, a_{3}\right)$ as shown in Figure 1. The values of $a_{2}$ and $a_{3}$ are computed from (6). We collect the result as follows:

Case 1a. Let $-1 / 2<\alpha<1,0 \leqq v \leqq k / 2+1$, and

$$
\begin{aligned}
& 2 a_{2}=(1-\alpha) v+\alpha(k / 2+1)+k / 2-1, \\
& 6 a_{3}=4 a_{2}^{2}+2\left(1-\alpha^{2}\right) v+\alpha^{2}(k+2)-k .
\end{aligned}
$$

The maximum of $a_{4}$ is then given by (8), where $\max G=4 \alpha^{2}, \min G=-4 \alpha^{2}-16 \alpha-8$, and $t, u$ are given by (10). 
The value $\alpha=1$ belongs to the function $f_{k}$ of (4) generated by the measure with $\mu^{+}=(k / 2+1) \delta(0)$ and $\mu^{-}=(-k / 2+1) \delta(\pi)$. For this function the formula (8) yields the global maximum $\left|a_{4}\right|=\left(k^{3}+8 k\right) / 24$ [6].

Suppose next that $\alpha=-1 / 2$. Now $t=u=0$ and the points of (8) satisfy $\cos \varphi_{1}=-1 / 2, \cos \varphi_{2}=1 / 2$. The maximum of $G$ occurs at $\varphi=0$ and $\varphi= \pm \varphi_{1}$, the minimum at $\varphi=\pi$ and $\varphi= \pm \varphi_{2}$, so the generating $\mu$ consists of the measures $v \delta(0),(1 / 2)(k / 2+1-v) \delta\left( \pm \varphi_{1}\right)$, and $-v^{\prime} \delta(\pi),(-1 / 2)\left(k / 2-1-v^{\prime}\right) \delta\left( \pm \varphi_{2}\right)$, where $0 \leqq v \leqq k / 2+1,0 \leqq v^{\prime} \leqq k / 2-1$. The formula (8) assumes a simple form, and we can state the result:

Case 2. Let $0 \leqq v \leqq k / 2+1,0 \leqq v^{\prime} \leqq k / 2-1$, and let the coefficients $a_{2}, a_{3}$ be given by

$$
\begin{aligned}
& 2 a_{2}=(3 / 2)\left(v+v^{\prime}\right)-k / 2, \\
& 6 a_{3}=4 a_{2}^{2}+(3 / 2)\left(v-v^{\prime}\right)-1 .
\end{aligned}
$$

The sharp upper bound for $a_{4}$ is

$$
a_{4} \leqq(3 / 2) a_{2} a_{3}-(2 / 3) a_{2}^{3}+k / 12 .
$$

This inequality can of course also be obtained directly from (6).

Let $-1<\alpha<-1 / 2$. The order of the expressions (11) becomes $G(0)=G\left( \pm \varphi_{1}\right)>$ $G(\pi)>G\left( \pm \varphi_{2}\right)$. Accordingly, the extremal $\mu$ consists of the five parts $v \delta(0)$, $(1 / 2)(k / 2+1-v) \delta\left( \pm \varphi_{1}\right)$, and $(-1 / 2)(k / 2-1) \delta\left( \pm \varphi_{2}\right)$, where $0 \leqq v \leqq k / 2+1$. As in Case 1a, the measure $\mu$ determines the boundary of $\left(a_{2}, a_{3}, a_{4}\right)$ :

Case 3a. Let $-1<\alpha<-1 / 2$ and $0 \leqq v \leqq k / 2+1$. If the coefficients $a_{2}, a_{3}$ are given by

$$
\begin{gathered}
2 a_{2}=(1-\alpha) v+\alpha(k / 2+1)-(1 / 3)(\alpha+2)(k / 2-1), \\
6 a_{3}=4 a_{2}^{2}-2+2\left(1-\alpha^{2}\right) v+\alpha^{2}(k+2)-(1 / 9)(\alpha+2)^{2}(k-2),
\end{gathered}
$$

the maximum of $a_{4}$ is calculated from (8), where $\max G=4 \alpha^{2}, \min G=4(\alpha+2)^{2}$. $(4 \alpha-1) / 27$, and $t, u$ are as in (10).

Choose next

$$
\begin{aligned}
& t=-2(2 \alpha-1) \\
& u=4 \alpha^{2}-8 \alpha+3,
\end{aligned}
$$

$-1 \leqq \alpha \leqq 1$. The expression (9) assumes the values $\cos \varphi_{1}=\alpha, \cos \varphi_{2}=(\alpha-2) / 3$, and it follows that

$$
\begin{aligned}
G\left( \pm \varphi_{1}\right) & =G(\pi)=-4 \alpha^{2}, \\
G\left( \pm \varphi_{2}\right) & =4(\alpha-2)^{2}(4 \alpha+1) / 27, \\
G(0) & =4 \alpha^{2}-16 \alpha+8 .
\end{aligned}
$$


These numbers satisfy $G(0)>G\left( \pm \varphi_{2}\right)>G\left( \pm \varphi_{1}\right)=G(\pi)$ for $-1<\alpha<1 / 2$, and $G\left( \pm \varphi_{2}\right)>G(0)>G\left( \pm \varphi_{1}\right)=G(\pi)$ for $1 / 2<\alpha<1$. The conclusions are completely analogous to those in Cases 1a and $3 \mathrm{a}$, and we merely describe the extremal $\mu$.

Case 1b. For $t, u$ given by (14), $-1<\alpha<1 / 2$, the maximum of $a_{4}$ occurs when the support of the measure $\mu$ consists of four points. The positive part of $\mu$ is concentrated on $\varphi=0$, the negative part is distributed among the point $\varphi=\pi$ and the points where $\cos \varphi=\alpha$.

Case 3b. For $t, u$ given by (14), $1 / 2<\alpha<1$, the maximum of $a_{4}$ occurs when the support of $\mu$ consists of five points. The positive part of $\mu$ is distributed among the points where $\cos \varphi=(\alpha-2) / 3$, the negative among the points $\varphi=\pi$ and $\cos \varphi=\alpha$.

Note that one obtains the measures in the $b$-cases from those of the $a$-cases by rotating the points of spt $\mu$ by $\pi$ and changing the roles of $\mu^{+}$and $\mu^{-}$.

The value $\alpha=-1$ in Case 3a implies $\max G=G(0)=G(\pi)$ and $\min G=G(\varphi)$, where $\cos \varphi=1 / 3$. The condition $G(0)=G(\pi)$ is turn gives $u=-1$, but allows the parameter $t$ to be arbitrary. This case is also contained in the following choice of parameters.

Let

$$
\begin{aligned}
& t=1 / \alpha-3 \alpha, \\
& u=-1
\end{aligned}
$$

$0<\alpha \leqq 1 / 3$. The only admissible points calculated from (9) are given by $\cos \varphi_{1}=\alpha$. Now $G\left( \pm \varphi_{1}\right)=-2 \alpha\left(\alpha^{2}+1\right)$ and $G(0)=G(\pi)=2 / \alpha-6 \alpha$, so the maximum occurs at $\varphi=0$ and $\varphi=\pi$, the minimum at $\varphi= \pm \varphi_{1}$. The positive part of $\mu$ consists of the measures $v \delta(0)$ and $(k / 2+1-v) \delta(\pi)$, the negative of $(-1 / 2)(k / 2-1) \delta\left( \pm \varphi_{1}\right)$. The expression (8) can be slightly simplified, and we arrive at the following formulae:

Case 4 a. Let $0<\alpha \leqq 1 / 3,0 \leqq \nu \leqq k / 2+1$. If the coefficients $a_{2}, a_{3}$ are given by

$$
\begin{aligned}
& 2 a_{2}=2 v-(k / 2+1)-\alpha(k / 2-1), \\
& 6 a_{3}=4 a_{2}^{2}+k-\alpha^{2}(k-2),
\end{aligned}
$$

the sharp upper bound for $a_{4}$ is

$$
a_{4} \leqq\left(-4 a_{2}^{3}+9 a_{2} a_{3}+a_{2}\right) / 6+(1 / 3)\left(\alpha-\alpha^{3}\right)(k / 2-1) .
$$

Case 4b. Choose $t, u$ as in Case 4 a but take $\alpha$ in the interval $-1 / 3 \leqq \alpha<0$. The result is an extremal $\mu$ whose positive part is concentrated on the points where $\cos \varphi=\alpha$, the negative part on $\varphi=0$ and $\varphi=\pi$. We omit the details.

Finally, the remaining parts of $\left(a_{2}, a_{3}\right)$ will be covered by

$$
\begin{aligned}
& t=\mp 3(x+y) \\
& u=12 x y+3 .
\end{aligned}
$$


The formula (9) implies for the support point candidates $\cos \varphi_{1}= \pm x, \cos \varphi_{2}= \pm y$. For these points we choose the representations

$$
\begin{aligned}
& x= \pm(1+\beta) / 2, \\
& y= \pm(4 \alpha \beta-3 \beta-3) / 6,
\end{aligned}
$$

where $0 \leqq \alpha, \beta \leqq 1$.

Take the minus sign in (15) (plus in (16)). At the possible points of spt $\mu$ we have

$$
\begin{aligned}
G\left( \pm \varphi_{1}\right) & =(1+\beta)^{2}(-1-\beta+\alpha \beta), \\
G\left( \pm \varphi_{2}\right) & =(3+3 \beta-4 \alpha \beta)^{2}(3+3 \beta-\alpha \beta) / 27 \\
G(0) & =4 \alpha \beta^{2}-3 \beta^{2}-6 \beta+1, \\
G(\pi) & =-4 \alpha \beta^{2}-8 \alpha \beta+3 \beta^{2}+6 \beta-1 .
\end{aligned}
$$

It is not difficult to check that for all $0 \leqq \alpha, \beta \leqq 1$ the maximum of $G$ occurs at the points $\varphi= \pm \varphi_{2}$, the minimum at $\varphi= \pm \varphi_{1}$. It follows from (16) that $\cos \varphi_{2}$ runs the interval $-\cos \varphi_{1} \leqq \cos \varphi_{2} \leqq\left(\cos \varphi_{1}-2\right) / 3$ for every $\cos \varphi_{1} \in[1 / 2,1]$. The support of the extremal $\mu$ consists of four points, and $\mu$ has equal positive and equal negative values at the respective points. We have:

Case 5a. Let $0 \leqq \alpha, \beta \leqq 1$, and let the coefficients $a_{2}, a_{3}$ be given by

$$
\begin{aligned}
& 2 a_{2}=\frac{4 \alpha \beta-3 \beta-3}{6}\left(\frac{k}{2}+1\right)-\frac{1+\beta}{2}\left(\frac{k}{2}-1\right), \\
& 6 a_{3}=4 a_{2}^{2}+\frac{(4 \alpha \beta-3 \beta-3)^{2}}{18}\left(\frac{k}{2}+1\right)-\frac{(1+\beta)^{2}}{2}\left(\frac{k}{2}-1\right)-2 .
\end{aligned}
$$

The maximum of $a_{4}$ is obtained by (8), where $t, u$ are given by (15) and (16), and $\max G=G\left( \pm \varphi_{2}\right), \min G=G\left( \pm \varphi_{1}\right)$ by (17).

Case 5b. Take the plus sign in (15). This effects merely a change of signs in (17). So, if $\max G$ and $\min G$ have the same values as in Case 5a, we see that the maximum of $G$ equals $-\min G$, the minimum $-\max G$, and these values occur at $\varphi= \pm \varphi_{1}$ and $\varphi= \pm \varphi_{2}$, respectively. The boundary of $\left(a_{2}, a_{3}, a_{4}\right)$ is computed as in 5a.

All the points of $\left(a_{2}, a_{3}\right)$ are covered in a unique manner in the foregoing Cases $1 \mathrm{a}-5 \mathrm{~b}$ and the maximal $a_{4}$ has been found for each pair $\left(a_{2}, a_{3}\right)$. The minima of $a_{4}$ are obtained by the rotation $f(z) \rightarrow-f(-z)$, which transforms the point $\left(a_{2}, a_{3}, a_{4}\right)$ into $\left(-a_{2}, a_{3},-a_{4}\right)$. (Equivalently, one can place the positive values of $\mu$ at the points where the function $G$ reaches its minimum and the negative values at the maximum points.)

For any boundary point of $\left(a_{2}, a_{3}, a_{4}\right)$ we get the extremal function $f$ from (5) by substituting the corresponding extremal measure $\mu$ (we omit here the rather lengthy expressions). These Schwarz-Christoffel mappings take the unit disc onto polygons with at most six corners (or sides). There are at most three vertices where the line segment turns to the left, and at most three vertices where it turns to the 
right. We see that all the possibilities concerning the number of both kinds of vertices indeed occur.

In Figure 2 the surface $\left(a_{2}, a_{3}, \max a_{4}\right)$ in central projection is shown in the case

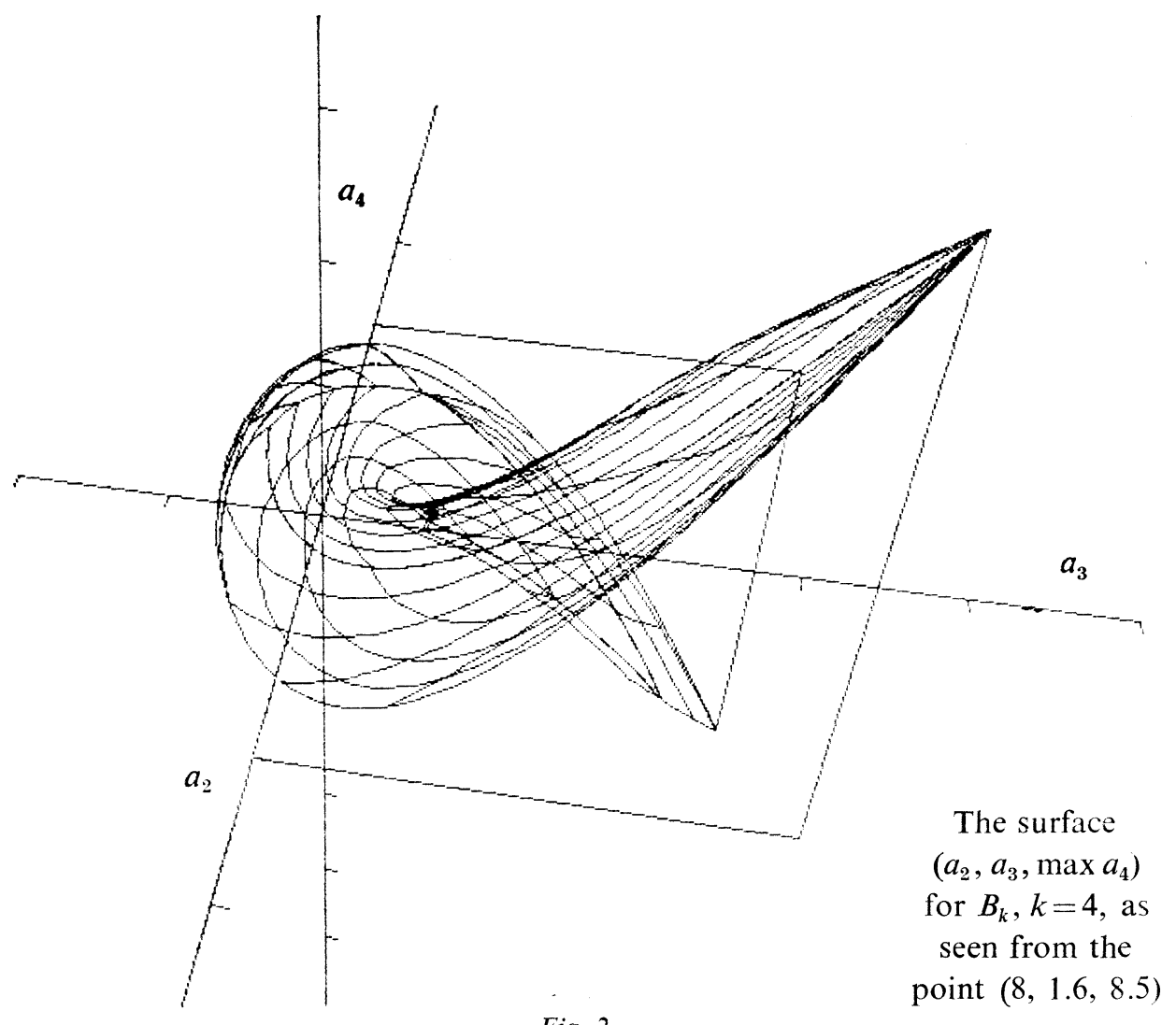

Fig. 2.

$k=4$. We are thankful to Prof. S. Mustonen for providing us with the program and facilities needed in drawing the pictures.

\section{On the inverse coefficients}

Denote by $W_{k}$ the class of functions inverse to functions in $B_{k}$. Let $F_{k}=f_{k}^{-1}$ and for $F \in W_{k}$ write

$$
\begin{gathered}
F(w)=w+\sum_{n=2}^{\infty} A_{n} w^{n}, \\
F_{k}(w)=w+\sum_{n=2}^{\infty} A_{n}(k) w^{n} .
\end{gathered}
$$


If $F \in f^{-1}$, where $f$ is given by (1), we have

$$
\begin{aligned}
& A_{2}=-a_{2}, \\
& A_{3}=-a_{3}+2 a_{2}^{2}, \\
& A_{4}=-a_{4}+5 a_{2}\left(a_{3}-a_{2}^{2}\right) .
\end{aligned}
$$

The bound $\left|A_{2}\right| \leqq A_{2}(k)=k / 2$ follows immediately. In [4] Kirwan and Schober showed that $\left|A_{3}\right| \leqq A_{3}(k)$ for all $k \geqq 2$, and $\left|A_{4}\right| \leqq A_{4}(k)$ for $k \geqq 2 \frac{1}{3}$. In both cases the extremum is reached by the function $F_{k}$ (or by a rotation of it). On the other hand, it can be seen, cf. [4], that the function $F_{k}$ is not extremal for $A_{n}$ with larger values of $n$ (for instance with $n=10$ ), if $k$ is sufficiently close to 2 . Thus it might have been expected that $F_{k}$ was not extremal for $A_{4}$ for some values $2<k<2 \frac{1}{3}$. However, we shall see that $A_{4}$ is maximized by $F_{k}$, at least in the real case.

Since we have the parametrical representations for the boundary of $\left(a_{2}, a_{3}, a_{4}\right)$ in the class $B_{k}$, the formulas (18) give the corresponding parametrizations for the boundary of $\left(A_{2}, A_{3}, A_{4}\right)$ of $W_{k}$. Thus the formulae in the preceding chapter together with (18) in fact give the coefficient body $\left(A_{2}, A_{3}, A_{4}\right)$ of $W_{k}$ in the real case.

Now we can compute the maximum of $\left|A_{4}\right|$ for any $k \geqq 2$. Because of the rotation $F(w) \rightarrow-F(-w)$ it suffices to find the minimum of $A_{4}$. We do this by minimizing the expression (18) of $A_{4}$ in all the different parts of the boundary of $\left(a_{2}, a_{3}, a_{4}\right)$ and picking the smallest among these local minima. For each part we substitute in (18) the expressions of $a_{2}, a_{3}$ and of the maximal $a_{4}$ in terms of the given parameters. This leads to polynomials of third degree in two parameters. For example, in (the simplest) Case 2 a substitution of the formulas (12) and (13) into the expression of $A_{4}$ yields the polynomial

$=\frac{k^{3}+2 k}{32}-\frac{14+9 k^{2}}{32}\left(v+v^{\prime}\right)+\frac{7 k}{32}\left(v^{\prime}-v\right)+\frac{27}{32} k\left(v+v^{\prime}\right)^{2}+\frac{21}{32}\left(v^{2}-v^{\prime 2}\right)-\frac{27}{32}\left(v+v^{\prime}\right)^{3}$ $0 \leqq v \leqq k / 2+1,0 \leqq v^{\prime} \leqq k / 2-1$. A straightforward computation shows that for $k \geqq 2$ the minimum of the polynomial is reached with the values $v=k / 2+1, v^{\prime}=$ $k / 2-1$, i.e., with the function $F_{k}$. Accordingly, the maximum occurs with the function $-F_{k}(-w)$.

In a similar manner we could produce the polynomials of $A_{4}$ in the remaining cases. However, the expressions are more involved and the computations become exceedingly lengthy. Under these circumstances we were glad to leave the minimization to the computer. A standard minimum finding program was applied to each of the Cases 1a, 1b, 3a, 3b, 4a, 4b, 5a, 5b. In Cases $1 \mathrm{a}$ and $1 \mathrm{~b}$ the minimum occurred for all tested $k \geqq 2$ with the parameter values giving the function $F_{k}$. In all the other Cases $3 \mathrm{a}-5 \mathrm{~b}$ the minimum was clearly larger than the value $-k\left(k^{2}-2\right) / 4$ given 
by $F_{k}$. The situation is visualized in Figure 3 , where the surface $\left(A_{2}, A_{3}, \max A_{4}\right)$ is presented for $k=2 \frac{1}{4}$.

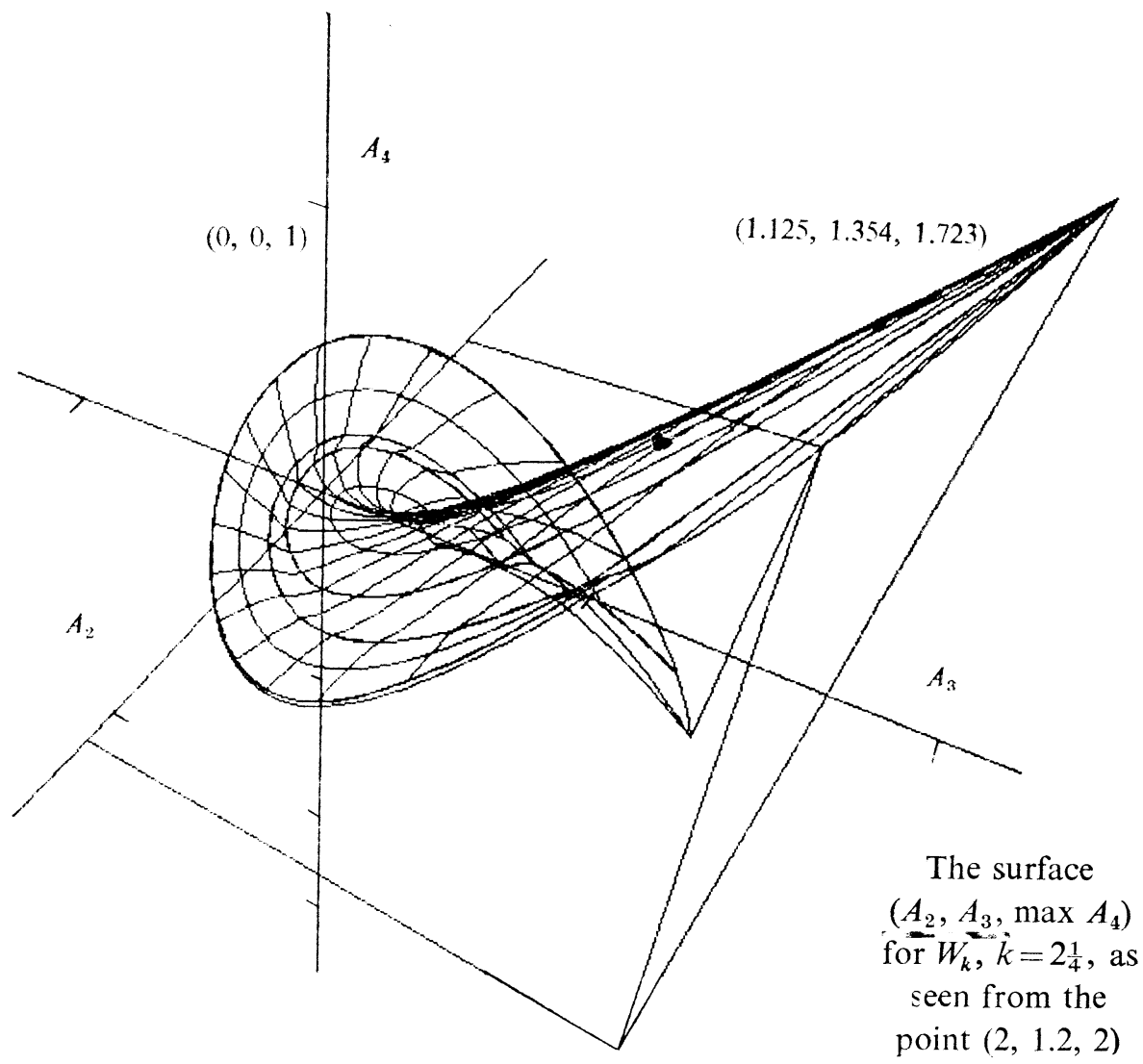

Fig. 3.

On the basis of the numerical evidence we can state the following conclusion: If $F \in W_{k}, F(w)=w+A_{2} w^{2}+\ldots$, where the coefficients $A_{2}, A_{3}, A_{4}$ are real, then

$$
\left|A_{4}\right| \leqq \frac{k\left(k^{2}-2\right)}{4}
$$

for all $k \geqq 2$. Equality holds only if $F(w)=\tau F_{k}(\tau w)$ with $\tau= \pm 1$. 


\title{
References
}

[1] Aharonov, D., and S. Friedland: On an inequality connected with the coefficient conjecture for functions of bounded boundary rotation. - Ann. Acad. Sci. Fenn. Ser. A I Math. 524, 1972, 1-14.

[2] Brannan, D. A., J. G. Clunie, and W. E. Kirwan: On the coefficient problem for functions of bounded boundary rotation. - Ibid. 523, 1973, 1-18.

[3] HaArio, H.: On the coefficient bodies of univalent functions. - Ann. Acad. Sci. Fenn. Ser. A I Math. Dissertationes 22, 1978, 1-49.

[4] Kirwan, W. E., and G. Schober: Inverse coefficients for function of bounded boundary rotation. - J. Analyse Math. 36, 1979, 167-178.

[5] Pfluger, A.: Functions of bounded boundary rotation and convexity. - Ibid. 30, 1976, $437-451$.

[6] Schiffer, M., and O. TAMmI: On the fourth coefficient of univalent functions with bounded boundary rotation. - Ann. Acad. Sci. Fenn. Ser. A I Math. 396, 1967, 1-26.

[7] TAмmi, O.: Extremum problems for bounded univalent functions. - Lecture Notes in Mathematics 646, Springer-Verlag, Berlin-Heidelberg-New York, 1978.

\author{
University of Helsinki \\ Department of Mathematics \\ SF-00100 Helsinki 10
}

Finland

Received 16 October 1981 